A N N A L E S Annales de Bretagne et des Pays de l'Ouest

Anjou. Maine. Poitou-Charente. Touraine

109-1 | 2002

Varia

\title{
Marie-Thérèse CLOITRE (dir.), Histoire de Brest
}

\section{François Chappe}

\section{OpenEdition}

Journals

Édition électronique

URL : http://journals.openedition.org/abpo/1662

DOI : $10.4000 /$ abpo. 1662

ISBN : 978-2-7535-1485-0

ISSN : 2108-6443

Éditeur

Presses universitaires de Rennes

Édition imprimée

Date de publication : 20 mars 2002

Pagination : 181-187

ISBN : 978-2-86847-708-8

ISSN : 0399-0826

Référence électronique

François Chappe, "Marie-Thérèse CLOITRE (dir.), Histoire de Brest », Annales de Bretagne et des Pays de I'Ouest [En ligne], 109-1 | 2002, mis en ligne le 20 mars 2004, consulté le 23 septembre 2020. URL : http://journals.openedition.org/abpo/1662 ; DOI : https://doi.org/10.4000/abpo.1662 
Il est peu probable que les auteurs de l'introduction, de la préface et du premier chapitre de cette histoire de Brest aient comparé les premiers mots de leurs textes respectifs. Y. Le Gallo évoque un " ouvrage collectif récent ", J.-P. Pinot affirme que "l'histoire d'une ville, ce sont les états successifs de sa géographie ", P. Galliou et J.-Y. Éveillard méditent sur " le territoire qu'occupe aujourd'hui l'agglomération brestoise ". Même non-concerté, le choix de ces " entames " suggère l'ambition interdisciplinaire qui a animé les auteurs de ce nouvel ouvrage collectif, dirigé par M.-T. Cloître dont l'équipe a été confrontée à trois exigences inhérentes à ce type de projet : combiner les données d'une histoire singulière avec celles des structures politiques, économiques, culturelles dans lesquelles la ville étudiée s'incorpore successivement; féconder à l'intérieur de cette histoire singulière les apports des diverses sciences sociales concernées par toute histoire de ville qui est en même temps celle d'un ensemble d'approches obligatoirement diversifiées, elles-mêmes incorporées à des problématiques variables en fonction des périodes étudiées; raconter enfin une histoire qui est celle de Brest et d'aucune autre ville.

Quelques éventuelles frustrations mineures n'altèrent pas le bonheur de lecture que procure cet ouvrage qui a relevé ces trois défis avec rigueur, brio, voire avec élégance. Une expression moins imprudente qu'invérifiable : «Pour Brest, plus peut-être que pour d'autres villes, les conditions géographiques [...] relèvent de deux groupes de données : le site, la situation " (p. 15), l'abus de l'usage du mot " vocation" (p. 5, 6, 7, 47, 49, 75, 181, 268 etc.), prouvent que les historiens sont toujours soumis à la tentation du péché de téléologie. Il est souvent commis dans les histoires dites maritimes, "la proximité de l'océan faisait que Lorient, Le Havre, Marseille ne pouvaient que etc. ». Nos auteurs n'y ont pas succombé s'obligeant au croisement permanent entre les facteurs locaux de l'histoire de Brest et les conditions globales qui ont favorisé l'efficacité des premiers, P. Jarnoux, Y. Le Gallo, F. Roudaut ont clairement fixé ce refus d'un quelconque déterminisme local au profit de l'étude d'une active attention portée de l'extérieur à ces réalités locales : «Brest qui, à l'origine, ne semble pas plus privilégié que les autres ports du Ponant, devient l'objet d'un intérêt croissant et s'impose comme la base navale primordiale " (p. 78).

C'est l'érudition des auteurs qui commande la prudence avec laquelle est rédigé le premier chapitre : "Aux origines de Brest. " Cette prudence prospective qui est l'opposé d'une quelconque frilosité leur permet d'insérer le territoire de Brest pendant l'âge de fer et après la conquête romaine dans une "économie ouverte" (p. 22). Les méticuleuses observations sur le castellum romain procèdent aussi d'une prudence méthodologique qui n'interdit pas de suggérer l'incorporation de ce bâtiment dans une organisation défensive globale, ellemême soumise aux aléas de la vie de l'Empire.

Avant que R. Le Prohon et J. Tanguy n'établissent le constat de la « Naissance d'une ville (XVI $-\mathrm{XVIII}{ }^{\mathrm{e}}$ siècles) ", B. Tanguy et J.-C. Cassard ont pertinemment souligné le caractère potentiel des dispositions géographiques et géopolitiques de Brest : «Brest n'existe pour l'heure qu'en négatif, telle une coquille vide à garder par précaution sans en avoir l'usage immédiat " (p. 39), analyse confirmée à la fin de ce même chapitre, "Brest au Moyen Âge ", par l'heureuse formule " $\mathrm{Au}$ $\mathrm{XV}^{\mathrm{e}}$ siècle, une ville à naître ". Ces virtualités se sont concrétisées en fonction d'événements, de choix, d'initiatives qui sont constamment analysés avec une méthode rien moins que déterministe, les auteurs paraissant s'être inspirés de la clef d'écriture, comme on dit une clef en musique, proposée dès l'introduc- 
tion à propos des causes de l'alternance entre périodes européennes maritimes et terriennes qui " ne sont pas géographiques : " c'est l'évolution de la société européenne qui dicte le passage parfois brutal d'un type à l'autre " (p. 17).

Si la singularité maritime, commerciale et militaire de Brest ne se dément pas, cette ville se dilate ou se contracte, s'épanouit ou s'anémie en fonction des événements militaires français (guerres civiles pendant la Ligue, la Révolution), européens (présence anglaise au XIV e siècle; " à partir des années 1660, Brest vit au rythme de la guerre et de la paix en Europe " (p. 75), tenant compte des conjonctures économiques et commerciales (reprise du début du XvII ${ }^{\mathrm{e}}$ siècle [p. 66]); géopolitiques (mouvement d'exploration fin XvII ${ }^{\mathrm{e}}$ siècle), directement influencée qu'elle est par les choix politiques effectués par l'État (lettres patentes d'Henri IV, bienveillante attention de Richelieu, initiative rationnelle et déterminée de Colbert, inventeur de l'Arsenal, relâchement du pouvoir royal au début du XVIII ${ }^{\mathrm{e}}$ siècle, relance de l'effort par Choiseul, interventionnisme idéologique et policier de Jean bon Saint-André).

À la suite de " la plongée dans l'abîme entre 1799 et 1815 ", analysée par J.-Y. Carluer, l'exigence de la compréhension de ce va et vient entre le local, le national, le mondial reste entière. Même si le chapitre "Les derniers temps de Brest " colonie " (1815-1848) " est centré sur la vie intérieure de Brest qui " continuait de perpétuer la cité du XvIII ${ }^{\mathrm{e}}$ siècle " (p. 155), Y. Le Gallo souligne la dépendance de Brest vis-à-vis des fluctuations politiques nationales (épisode des Cent Jours) et son incorporation aux théâtres maritimes méditerranéen (expédition d'Alger) et mondial (navigation entre Brest et la Martinique).

Le titre du chapitre rédigé par M.-T. Cloître « Première préfecture maritime et Marseille de l'Océan " suggère que le " destin impalpable " (p. 47) de Brest au Moyen Âge connaît son accomplissement. Même si les réalisations ne sont pas nécessairement à la hauteur des ambitions énoncées localement, par exemple par l'ingénieur Vincent souhaitant que Brest devienne " un vaste entrepôt de marchandises du monde entier, un grand chantier de réparation et un centre de ravitaillement de navires " (p. 163), "l'élan des armées impériales où l'amélioration de la conjoncture économique et sociale coïncidant avec les vues de Napoléon III amène à croire en Brest ville d'avenir, à son nouveau port de commerce, au port de guerre rénové " (p. 175). Brillantes perspectives laminées par "le temps du désenchantement, 1866-1870 " (p. 175) avant que ne vienne celui des " convulsions " accompagnant " la recherche d'un équilibre, 1870-1918 ". Dans ce chapitre, Y. Le Gallo décrit " la cité rouge " (p. 179) mais il souligne pertinemment que la ville maritime, la cité bleue, vit encore dans la dépendance de choix, d'arbitrages annihilant les grandes visions et espérances des défenseurs du port commercial breton, comme le fils de l'ancien Président de la République, Claude Casimir Périer, persuadé que l'opinion publique française « ne trouvera ni au-dessous de nos ambitions, ni au-dessus de notre pouvoir la conquête de l'Atlantique et l'hégémonie commerciale du monde " (p. 199). La Première Guerre mondiale démontra les immenses capacités nautiques de la rade de Brest mais une fois encore, en phase avec la conjoncture internationale, le destin de Brest fut celui d'une retombée dans l'atonie.

Analysant les mythes et les réalités du " vieux Brest " (1919-1944), Yvon Tranvouez continue d'appréhender les conditions de l'emboîtement de l'histoire de Brest dans celle de l'Europe en pointant par exemple sa dépendance vis-à-vis du choix des sociétés pétrolières qui préfèrent installer à Donges leurs complexes de traitement des hydrocarbures en même temps qu'échoue un projet de port aérien transatlantique. Si les commandes de la Marine atténuent les effets de la crise mondiale, il n'en reste pas moins que par rapport au transport 
des voyageurs ou des marchandises "Brest n'est ni une destination, ni un point de départ, simplement un lieu de transit dont chacun aspire à s'affranchir " (p. 213). L'enjeu que constitue Brest pendant la Seconde Guerre mondiale est clairement exposé ainsi que les choix stratégiques effectués pendant la campagne de libération.

Traitant la période courant de cette dernière à nos jours, E. Monange ne modifie pas un regard constamment fixé sur la ligne bleue du monde. Retour d'une relative prééminence de la Marine nationale dont les choix stratégiques influencent directement le développement de Brest, choix de l'expansion du secteur tertiaire en fonction d'un désenclavement enfin effectué, perspectives créées en fonction des politiques plus ou moins affirmées d'aménagement du territoire, dessinent, après l'impérieuse nécessité de " revivre ", le ferme espoir de " changer " les conditions d'une " affirmation " de Brest selon la belle formule d'H. Queffélec : " Le Brest énergiquement actuel invite à réfléchir de bonne foi aux nuits du passé. " E. Monange synthétise avec bonheur les obligations respectives pour l'historien de comprendre Brest dans le monde et pour les acteurs brestois d'être conscients des conséquences de ces nécessaires ouvertures : " Ouverture sur ce pays de Brest qui vient de naître... sur le Finistère... sur la Bretagne... sur la France... sur l'Europe... sur le monde " (p. 287).

Bref, le premier défi consistant à expliquer que Brest est dans Brest et dans le monde a été relevé avec bonheur. Le deuxième défi, l'ambition de multiplier les points de vue tout en les inscrivant dans des problématiques comparables tout au long des diverses périodes de cette histoire de Brest a produit des résultats très probants. Ainsi un lien permanent est établi entre la démographie, l'urbanisme, les conditions de vie, lien qui tisse une histoire sociale constamment inscrite sur un territoire. R. Leprohon et Jean Tanguy inaugurent, pour les Xvl $\mathrm{e}^{\mathrm{e}}$ et XVII ${ }^{\mathrm{e}}$ siècles, cette méthode fructueuse (on comprend aisément qu'elle ne pouvait être mise en œuvre pour les périodes précédentes). Ainsi la révolution démographique qui s'est produite au XvII ${ }^{\mathrm{e}}$ siècle, pertinemment constatée dès

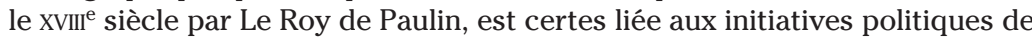
Colbert mais est aussi inscrite dans un cercle de compréhension incluant l'urbanisme pour partie reflet des conditions de travail (aménagement des venelles de Keravel), " les préoccupations en matière de voirie et d'hygiène " (p. 53), les migrations suscitées par la fiscalité et le va et vient entre ville et campagne. Les auteurs du chapitre consacré au XVIII siècle continuent de lier contraintes spatiales, développement démographique, conditions de vie quotidienne, en repérant la répartition géographique des hommes selon leurs fonctions économiques et militaires. Du côté des défavorisés, l'évocation du quartier du Pont de Terre est particulièrement suggestive et colorée (p. 102).

Cette cohérence est maintenue au cours des chapitres suivants. Si les chiffres de la population brestoise sont instructifs en eux-mêmes (Restauration et Monarchie de Juillet, p. 151; Second Empire, p. 107; III République d'avant 1914, p. 195; entre deux guerres, p. 223; après guerre, p. 281), leur intérêt s'accroît par leur connexion aux préoccupations urbanistiques, portuaires, civiles et militaires évoquées pour ces mêmes périodes, p. 147, p. 170, p. 196 (III ${ }^{e}$ République d'avant 1914), p. 223, p. 281. La diversité des classes sociales brestoises incluant leurs répartitions spatiales est constamment soulignée : " une population composite " aux derniers temps de Brest " colonie " (1815-1848), (cf. p. 153); " les composantes sociales... " au début de la III République (p. 179), " les conflits sociaux " constatés au cours des vingt dernières années (p. 207) en même temps que sont appréhendés les facteurs de l'unité brestoise, le fait maritime en particulier bien que ce dernier ne soit ni exclusif, ni permanent, facteurs qui per- 
mettent d'écrire l'histoire d'" une " ville, volonté de synthèse illustrée par l'heureuse formule d'Yvon Tranvouez " unité et fractures de la société brestoise " (p. 222).

La même opportune symétrie entre l'approche des singularités et la saisie de leur incorporation dans un ensemble communautaire est repérable dans le domaine des mentalités, des cultures, des idéologies. À cause de la " pénurie documentaire " (p. 34), l'entreprise était délicate pour les auteurs de « Brest aux temps obscurs " (p. 29), mais J.-C. Cassard et B. Tanguy suggèrent cependant la densité du maillage des cadres paroissiaux. La présence, en surplomb, d'appareils idéologiques, politiques, religieux, culturels, qu'ils soient traditionnels (Église catholique, État) ou nouveaux (SFIO, anarcho-syndicalisme) est certes soulignée mais les auteurs de tous les chapitres se sont bien gardés de rédiger exclusivement une histoire de ces idéologies nationales ou internationales. Le choix permanent a été celui de la description de l'assimilation brestoise de ces idéologies, de ces innovations culturelles conférant ainsi un contenu concret au terme, souvent galvaudé, de "mentalités". Ainsi ont été marquées la précocité de l'implantation de la Franc-maçonnerie (p. 106), la singularité d'une culture maritime scientifique (p. 105) confinant à un exclusivisme dont la société civile brestoise eut du mal à s'émanciper (p. 146), la spécificité d'un anticléricalisme lié aux fluctuations idéologiques des cadres de la Marine (p. 144). Analysé avec une belle finesse par Y. Le Gallo, le paradoxe de l'antimilitarisme brestois sous la III République (p. 181) illustre la nécessité de croiser plusieurs types de données locales et universelles (haine du galon, refus de l'autorité...) pour appréhender la singularité du recyclage brestois d'une idéologie nationale, voire internationale. Par rapport à ce domaine comme par rapport à ceux précédemment évoqués (champs social, religieux, culturel, maritime) quelques comparaisons avec des villes de nature comparable (Cherbourg, Lorient, Rochefort, Toulon...) auraient peut-être été utiles.

Ces comparaisons auraient encore mieux souligné la singularité de cette " histoire " de Brest, le mot " histoire " renvoyant à la fois à la réalité de ce qui s'est passé à Brest et au compte rendu de cette dernière. Les auteurs ont équilibré leurs mises en perspective de Brest sous des horizons nationaux et internationaux, la multiplication de leurs points de vue maintenus en cohérence, tout au long des chapitres, par l'écriture d'une histoire qui s'est passée à Brest et nulle part ailleurs. L'attention aux individus est soutenue, humanisant ainsi une approche de structures jamais désincarnées. Ces individus sont évoqués dans la singularité de leurs itinéraires et de leur rayonnement personnel : Jacques Sané au XVIII ${ }^{\mathrm{e}}$ siècle (p. 93), l'abbé Graveran sous la Restauration et la Monarchie de Juillet (p. 143), le baron La Crosse sous Louis-Philippe (p. 146), P. Le Bris (p. 247), G. Lombard (p. 254) après la Seconde Guerre mondiale; les hommes sont saisis dans l'effervescence de leurs turpitudes financières (affaire Bellamy, p. 175), de leurs engagements dramatiques (mort du militant E. Mazé lors de la grève des ouvriers du bâtiment en 1950, p. 245) ou mélodramatiques (traque, en 1872, par l'intraitable Jean-Louis Kergrohen, des comportements coupables d'un " monsieur prêtre " et d' " une jeune veuve " dans le " train de nuit " reliant Châteaulin à Brest, p. 184). Les destinées sociales collectives sont aussi appréhendées dans leur spécificité brestoise. Si l'Arsenal a été étudié sous ses aspects politiques et techniques, le sort des hommes n'est jamais ignoré ("L'Arsenal et les problèmes ouvriers entre 1815 et 1848 ", p. 149; l'Arsenal comme foyer de tuberculose, p. 203) non moins que celui des bagnards et de leurs chiourmes. Le folklore local (voir le savoureux dialogue entre Francine et Soizik, p. 227) est évoqué avec une parcimonie qui évite l'or- 
nière de la facilité. Quant aux rythmes de travail à l'Arsenal ils sont suggérés avec une rare délicatesse d'écriture qui ménage simultanément la réalité historique et la dignité du travailleur, « cet employé de l'État [qui] dépense à des travaux supplémentaires l'énergie que le rythme paisible du travail à l'Arsenal lui permet d'économiser" (p. 182).

La qualité de l'écriture en général, de la présentation d'ensemble de l'ouvrage (témoignant de la compétence du maquettiste) permet une lecture facile, compatible avec une rigueur scientifique dont ont fait preuve les quatorze rédacteurs de cet ouvrage. Ce mérite, collectif, doit cependant être particulièrement souligné pour les auteurs des deux premiers chapitres, tenus d'effectuer constamment le déport entre hypothèses et résultats, pour celui du chapitre consacré à la Révolution et à l'Empire, tant le contexte idéologique chaud exigeait une honnêteté intellectuelle sans faille qu'a brillamment démontrée J.-Y. Carluer, pour E. Monange appréhendant, avec une chaleureuse objectivité, des données politiques " très contemporaines ".

Le lecteur non brestois peut être éventuellement décontenancé par l'extrême précision érudite avec laquelle sont détaillés les stratégies, alliances, résultats des élections municipales de 1904, législatives de 1910 et de 1925. Le lecteur brestois espérait peut-être plus de développement autour des questions scolaires entre les deux guerres. Quant à l'excellente problématique posée par le chapitre "Mythes et réalités du vieux Brest ", synthétisée par la brillante formule " [...] si le mythe s'enivre de mémoire, il se dessèche au contact de l'histoire " (p. 204), elle aurait peut-être pu encore s'enrichir par la levée d'un doute sur le sens du mot " mythe " : faut-il l'entendre exclusivement comme un pieux mensonge, une légende utile, un récit délibérément fabulateur? Ces réserves mineures, d'ailleurs elles-mêmes contestables, n'altèrent pas le bonheur d'une lecture, parachevé par une excellente bibliographie, classant et commentant sources primaires, ouvrages de synthèse, travaux de recherche, qui permet d'évaluer pour chaque période étudiée l'évolution des recherches et des résultats.

Il serait, en l'occurrence, injuste de s'en tenir à l'hommage rituellement rendu aux iconographies dont, trop souvent, le mérite est simplement d'exister. L'iconographie est variée, informative, émouvante, cohérente avec le texte. La disposition en vis-à-vis (p. 210-211) de deux photographies représentant respectivement " la sortie des ouvriers de l'Arsenal, porte de Carpon " et " les membres de la Chambre de commerce dans la grande salle des réunions, 1929 » témoigne du souci avec lequel l'iconographie s'est voulue significative. Les point de vue, les suggestions, les rapports se sont multipliés avec bonheur. Ainsi nombre de personnalités brestoises sont visualisables par le dessin (l'abbé Graveran, p. 145), la peinture (portrait de Sourdeac, p. 65), la sculpture (buste de Sané, p. 93), la caricature (baron La Crosse, p. 158), la photographie (les maires de Brest encore en vie lors de l'inauguration du pont de Recouvrance, p. 251; G. Lombard, p. 263).

Les groupes humains ont été saisis dans leurs fonctions collectives, religieuses (Bénédiction de l'établissement consacré aux pupilles de la Marine, 1863, p. 173), culturelles (fête bretonne à Brest en 1930, p. 227), politiques (photographies des municipalités Le Gorgeu et Maille, p. 219, 264), mondaines (garden party à la préfecture maritime, p. 181), sans qu'aient été occultées les scènes de la vie quotidienne à différentes époques (" sur la place de la Liberté dans les années 1900 "; représentation du marché par le peintre Piet, fin XIX ${ }^{\mathrm{e}}$ siècle; la rue de Siam en 1930), ni les témoignages des affrontements sociaux (grévistes de Brest chargés par les dragons en 1905, p. 188; pommes de terre déversées devant la mairie, 1992, p. 268). Sur différents supports (miniature, tableau, 
photo) les illustrations rappellent l'incorporation de Brest aux conflits européens et mondiaux (siège du château de Brest, p. 37; le combat du Vengeur, p. 127; les troupes américaines à Brest, p. 207; la capitulation allemande, p. 237; le centre de Brest en ruines, p. 238).

Les évolutions urbaines sont perceptibles, visibles grâce au choix de présenter des plans relief (p. 101, 104, 166) qui aident effectivement à comprendre l'urbanisation, des tableaux de peinture soulignant l'évolution de la répartition des espaces entre ville et campagne (gravure de Baugean sous la Restauration, p. 141 ; Le port de Brest de J.-C. Noël, 1846, p. 153), des photographies aériennes (Brest dans l'entre deux guerres, p. 225; Océanopolis et le port de plaisance, p. 276; les métamorphoses de la place de la Liberté, de la Libération à l'an 2000, p. 284-285; aujourd'hui, p. 8-9).

On aurait pu craindre une excessive focalisation de l'iconographie sur les fonctions militaires maritimes de Brest. Si ces dernières ont légitimement bénéficié de l'incorporation de photographies (le Charles de Gaulle avant sa mise à l'eau, p. 265, et à flot, p. 269), de plans (le port et l'Arsenal en 1767, p. 85, sous le Second Empire, p. 160), de tableaux (Le Foch et le Clemenceau de P. Le Merdy), de gravures sur bois (p. 62), d'illustrations de journaux (opportunément commentés, p. 199), l'activité maritime commerciale est largement suggérée par les plans (p. 165, 273), la photo (p. 198), la peinture (p. 208) ainsi qu'est évoquée l'activité patrimoniale (p. 276). Cette séparation entre les différentes fonctions maritimes de Brest n'a pas occulté le souci de représenter l'ensemble du port de Brest sur différents supports (la médaille, p. 56; le tableau de peinture, p. 72, $73,168,169)$ multipliant opportunément les points de vue : Brest vu du chenal, p. 88-89; vue prise " de l'intérieur " (p. 128); vue prise du parc aux vivres (p. 137138). Les auteurs des remerciements ont bien eu raison de mentionner, parmi les personnes et institutions qui ont contribué à la réussite de cette œuvre authentiquement collective le travail cartographique qui a été effectué, en plus de celui de maquettiste. Le CRCB qui a mené avec courage cette publication, n'a manifestement pas considéré a priori que l'émotion, l'affection, la nostalgie étaient les ennemis de la démarche scientifique. Il est certain que la " connaissance " de Brest, qui inclut la prise en compte de la représentation que les Brestois se font de leur ville, s'enrichit de connivences affectives exprimées de l'intérieur. Le regard produisant la splendide "Brest la blanche" (p. 248-249) ne peut être que celui d'un amoureux de Brest. Les éditeurs ont été inspirés en choisissant d'illustrer la jaquette par L'entrée de la rue de Siam, de ce même Pierre Péron, artiste emblématique de Brest. La préface d'Y. Le Gallo témoigne aussi des résultats que peut susciter l'amour d'une ville. Elle ne sacrifie à aucune des facilités que l'on peut redouter dans ce type d'exercice, celle de « l'essentialisation " de la ville étant la plus déplorable, mais le lecteur ne voit aucune contradiction entre les rigoureuses considérations historiques d'Y. Le Gallo et ce magnifique paragraphe qui est un poème d'amour dédié à " cette ville nouvelle ressurgie du passé " : "D'abord telle qu'elle est et telle qu'elle se perpétue, on y respire large et on y voit ample. Ce qui importe essentiellement, c'est son site marin et maritime de fin de continent qui la transfigurait lorsqu'elle n'était encore que champ de ruines. Située au bord d'un lac océanique chevauché par les tempêtes mais accueillante aux navires en détresse, elle est ceinturée de grandes beautés naturelles qui se déploient en cercles élargis et dont la pointe du compas pique sur Ouessant, cependant que les branches en balaient d'immenses espaces de terre et de mer d'une démesure dramatique " (p. 13).

Les commentaires publiés sur la quatrième de couverture procèdent souvent de l'autosatisfaction éditoriale. En l'occurrence, toute l'équipe animée par 
M.-Th. Cloître peut légitimement promouvoir ce remarquable ouvrage collectif en s'autorisant de la formule : "Bénéficiant d'une illustration abondante et largement rénovée, cette édition du Centre de Recherche Bretonne et Celtique est à la fois un beau livre et l'ouvrage de référence sur l'histoire de Brest à l'aube du troisième millénaire. "

François CHAPPE

CALIN, William, Minority Literatures and Modernism - Scots, Breton, and Occitan, 1920-1990, University of Toronto romance series, 2000, 399 p. [ISBN 0-80204836-6] (édition reliée) [ISBN 0-8020-8365-X] (format poche).

L'événement est suffisamment rare pour être souligné : les études d'ensemble sur les littératures dites " minoritaires " sont trop rares. L'ouvrage du Professeur William Calin vient donc à point pour combler en partie cette lacune. On peut bien sûr citer quelques ouvrages récents, mais malheureusement peu accessibles aux non-brittophones : Anke Simon, An emsav lennegel brezhonek hag an danevelloù berr ("Le mouvement littéraire de langue bretonne et le genre court "), Anna Mouradova, Kudennoù an treiñ diwar ar rusianeg e brezhoneg ("Les problèmes de traductions du russe au breton "), Jacqueline Gibson, Tem ar marv en oberennoù Per Denez (" Le thème de la mort dans l'œuvre de Per Denez ").

L'ouvrage de W. Calin repose sur l'étude et l'analyse de trois littératures " ouest-européennes " en langues minoritaires : celles de l'Écosse (aussi bien en gaélique qu'en scot), de la Bretagne et de l'Occitanie. Pour chacune de ces littératures W. Calin a pris en compte trois domaines, à savoir la poésie, le roman et le théâtre. Il a également circonscrit son étude à une période allant de 1920 à 1990 : c'est-à-dire d'une période directement issue du cataclysme que fut la première guerre mondiale jusqu'à une période " fin de siècle " (limite bien commode même si les mouvements littéraires se continuent au-delà). W. Calin a tenu également à limiter son étude à un certain nombre d'acteurs de ces littératures parce qu'ils ont été (ou sont encore) des acteurs phares de celles-ci.

Le maître-mot de l'ouvrage est bien "modernisme " et c'est par l'Écosse, la première chronologiquement à l'avoir intégré, que l'étude aborde le sujet. Hugh MacDiarmid est celui qui a renouvelé la littérature écossaise, auteur de A Drunk Man Looks at the Thistle et de To Circumjack Cencrasus.

Vient ensuite la Bretagne avec le travail de Roparz Hemon, fondateur et animateur du mouvement littéraire Gwalarn à partir de 1925. L'analyse du Prof. William Calin est particulièrement intéressante car elle se situe en dehors de toutes les polémiques qui ont eu cours ces derniers mois à propos de Roparz Hemon. Il en ressort que le travail littéraire (de toute une vie) de ce dernier doit être dissocié de son attitude pendant les trois années fatidiques de 19411944. La meilleure preuve, sans doute, est que l'un des autres auteurs analysés, Per-Jakez Helias, soit bien considéré comme un héritier de Gwalarn. Mais ici l'analyse se situe surtout au niveau du genre privilégié par chaque auteur étudié : la poésie pour Roparz Hemon, ainsi que pour Maodez Glanndour et Añjela Duval, le roman pour Youenn Drezen et Roparz Hemon, la nouvelle (genre court) pour Per Denez, et enfin la production théâtrale (extraordinaire) pour Per-Jakez Helias. 